\title{
Effect of delayed diagnosis and treatment of congenital hypothyroidism on intelligence and quality of life: an observational study
}

Aman Bhakti Pulungan, ${ }^{1}$ Myrte Everarda Oldenkamp, ${ }^{2}$ Adrianus Sarinus Paulus van Trotsenburg, ${ }^{2}$ Wiwik Windarti, ${ }^{1}$ Hartono Gunardi ${ }^{1}$

pISSN: 0853-1773 • elSSN: 2252-8083 https://doi.org/10.13181/mji.v28i4.3473 Med J Indones. 2019;28:396-401

Received: January 9, 2019

Accepted: August 29, 2019

\section{Authors' affiliations:}

${ }^{1}$ Department of Child Health, Faculty of Medicine, Universitas Indonesia, Cipto Mangunkusumo Hospital, Jakarta, Indonesia, 'Emma Children's Hospital, Amsterdam University Medical Centers, University of Amsterdam, Amsterdam, The Netherlands

\section{Corresponding author:} Aman Bhakti Pulungan

Department of Child Health, Faculty of Medicine, Universitas Indonesia, Cipto Mangunkusumo Hospital, Jalan Pangeran Diponegoro No. 71, Kenari, Senen, Central Jakarta 10430, DKI Jakarta, Indonesia

Tel/Fax: +62-21-3907742/

+62-21-3907743

E-mail: amanpulungan@mac.com

\begin{abstract}
BACKGROUND Congenital hypothyroidism is one of the most prevalent, albeit preventable causes of intellectual disability. This study determined the intellectual outcome and health-related quality of life (QoL) in children and adolescents clinically diagnosed with congenital hypothyroidism and the associations among age of starting thyroid hormone treatment, current free thyroxine (FT4) level, intelligence quotient (IQ), and QoL.
\end{abstract}

METHODS Intelligence (Wechsler scales) and QoL (PedsQLTM 4.0 parent proxy-report) tests were administered to 25 patients with congenital hypothyroidism in the pediatric clinic of Cipto Mangunkusumo Hospital, Jakarta. Nineteen patients underwent thyroid ultrasonography or scintigraphy to determine the etiology of congenital hypothyroidism.

RESULTS A total of 25 patients with the median age of 9 (5.5-12.5) years were recruited. Hemiagenesis of thyroid gland was the most common etiology. Eighteen patients had a total IQ of $<70$. The age of treatment initiation did not correlate with full scale IQ (FSIQ) $(r=-0.261, p=0.071)$ and verbal IQ (VIQ) $(r=-0.232, p=0.265)$. The late initiation of treatment was correlated with lower performance IQ (PIQ) $(r=-0.325, p=0.025)$. The higher current FT4 levels was correlated with higher scores of FSIQ $(r=0.314, p=0.046)$ and PIQ $(r=0.320, p=0.043)$. The late initiation of treatment $(r=0.006, p=0.980)$ and higher current FT4 levels $(r=0.246, p=0.310)$ were not correlated with QoL.

CONCLUSIONS The late initiation of thyroid hormone substitution in patients with congenital hypothyroidism negatively affects intellectual abilities.

KEYWORDS congenital hypothyroidism, etiology, intellectual disability, treatment, quality of life
Congenital hypothyroidism is one of the most prevalent causes of mental retardation. Nevertheless, the negative effects of thyroid hormone deficiency on the developing brain can be prevented through early detection of affected children by using newborn screening and initiating thyroxine treatment within the first 2 weeks of life. ${ }^{1}$ Hence, the diagnosis after the golden period of the first 2 weeks of life is considered as late diagnosis and treatment for congenital hypothyroidism.
Most developed countries had instituted routine newborn screening programs since the late 1970 s and early 1980s. ${ }^{2}$ Unfortunately, newborn screening has still not been established in Indonesia. Therefore, congenital hypothyroidism is still diagnosed late and often remains largely undetected. ${ }^{1}$ Despite the unavailability of actual data, the number of congenital diseases is estimated to be high, thereby advocating the need for nationwide newborn screening. The Ministry of Health Republic of Indonesia passed the newborn 
screening policy for congenital hypothyroidism in 2014. Even though the newborn screening program is now mandated, it is still not covered by the universal health coverage, which makes its implementation difficult, especially in remote areas.

Several studies have reported the relationship between time of treatment initiation and intellectual function in patients with congenital hypothyroidism. Before the establishment of newborn screening, Klein et $\mathrm{al}^{3}$ had reported patients treated before the age of 3 months showed better average IQ scores than patients treated after the age of 3 months. A systematic review in 2016 concluded that most studies concurred that treatment initiation in the first month of life was associated with more favorable outcomes, including intellectual development. ${ }^{4}$

The objective of this study was to assess the cognitive function and health-related quality of life (QoL) in children who were clinically diagnosed with congenital hypothyroidism in Indonesia. Although several previous studies had widely reported the association between cognitive function and delayed diagnosis and treatment of congenital hypothyroidism, this study is still significant to raise awareness on congenital hypothyroidism because newborn screening has not yet been established in Indonesia.,

\section{METHODS}

\section{Subjects}

At the time of the study, the newborn screening policy had not been passed and had not yet been implemented in Indonesia. The study recruited consecutively the congenital hypothyroidism patients who presented to the Pediatric Outpatient Clinic, Cipto Mangukusumo Hospital, Jakarta, Indonesia, from June to November 2014. IQ and QoL were measured during this period. In the absence of a proper medical filing system to track future appointments, patients or their caregivers had to be informed regarding the study protocol and enrolled in the study during their regular visits to the outpatient clinic.

The inclusion criteria were a diagnosis of congenital hypothyroidism and the current age being between 4 and 18 years. The exclusion criteria were signs or symptoms of any syndrome, such as Down syndrome or other genetic conditions, as well as a medical history of severe perinatal asphyxia. The diagnosis of congenital hypothyroidism was confirmed using serum thyroid function tests. In most patients, thyroid hormone treatment was started without any preliminary laboratory testing or thyroid imaging.

Informed consent was obtained from all parents or legal guardians. Notably, reaching caregivers for consent or follow-up was often a challenge owing to several Indonesians frequently changing their phone numbers. The researchers contacted the caregivers after the study was planned.

Patients had the right to quit the study at any time without consequences, and patients who discontinued the study were replaced by other patients. This study protocol was approved by the Ethical Committee of Cipto Mangunkusumo Hospital, which is linked to the Universitas Indonesia, Jakarta, Indonesia (No: 711/ H2.F1/ETIK/2013).

\section{Procedure}

All patients who met the inclusion criteria were included in the study. Patient data related to the date of birth, sex, gestational age, birth weight, birth length, age at thyroid hormone treatment initiation, growth and development history, signs and symptoms at presentation and before the diagnosis of congenital hypothyroidism, and history of previous diseases were obtained from the medical records. A complete physical examination, including height, weight, and head circumference, was performed during visits at Cipto Mangunkusumo Hospital after the study was planned. Also, FT4 and TSH levels recorded at the time of congenital hypothyroidism diagnosis were studied to determine the cause of congenital hypothyroidism. If the last laboratory result was older than 3 months, free thyroxine (FT4) and thyroid stimulating hormone (TSH) measurements were repeated to determine the current levels. Possible side effects of thyroid hormone treatment were recorded.

The method of intelligence quotient (IQ) measurement was age dependent. The Wechsler Preschool and Primary Scale of Intelligence for children under 7 years of age, the Wechsler Intelligence Scale for Children for children above 7 years of age, and the Wechsler-Bellevue Intelligence Scale questionnaire for children between the ages of 16 and 18 years were used. These three tests yielded a set of fullscale IQ (FSIQ), verbal IQ (VIQ), and performance IQ (PIQ) scores. A single psychologist administered all tests to avoid any interobserver bias. Furthermore, the psychologist was blinded to patients' medical 
Table 1. Characteristics of patients

\begin{tabular}{lc}
\hline Characteristics & $\mathrm{n}(\%)(\mathrm{N}=25)$ \\
\hline Female sex & $19(76)$ \\
\hline Age (years), median (min-max) & $9(5.5-12.5)$ \\
\hline Age at congenital hypothyroidism diagnosis & \\
\hline$\leq 1$ month & $1(4)$ \\
\hline$>1$ month & $24(96)$ \\
\hline Age at beginning of therapy & \\
\hline$\leq 1$ month & $1(4)$ \\
\hline$>1$ month & $24(96)$ \\
\hline Age when treatment began (months), & $18(0.96-108)$ \\
mean (range) & \\
\hline Presenting complaints at first diagnosis & $24(96)$ \\
\hline Developmental delay & $19(76)$ \\
\hline Feeding problems & $15(60)$ \\
\hline Constipation & $14(56)$ \\
\hline Umbilical hernia & $12(48)$ \\
\hline History of jaundice $>2$ weeks & $18(72)$ \\
\hline Total IQ score & $4(16)$ \\
\hline Normal (>90) & $2(8)$ \\
\hline Low average (80-89) & $1(4)$ \\
\hline Borderline (70-79) & \\
\hline Intellectual disability (<70) & \\
\hline
\end{tabular}

IQ=intelligence quotient

details. All patients had normal FT4 levels at the time of IQ testing, following the pediatric department's protocol.

Parents or legal guardians were asked to fill in the PedsQL ${ }^{T M} 4.0$ parent proxy-report according to the child's age range to measure QoL. The 23-item PedsQL ${ }^{\mathrm{TM}}$ Generic Core Scales were designed to measure the core dimensions of health as delineated by the World Health Organization, as well as role (school) functioning. The four multidimensional scales consisted of physical, emotional, social, and school functioning. Subsequently, thyroid ultrasound and scintigraphy examinations were performed in those patients whose parents or legal guardians agreed for further investigations into the etiology of the congenital hypothyroidism.

\section{Statistical analysis}

Statistical analyses were performed using SPSS, version 22 (IBM). Correlations between variables were measured using Kendall's Tau correlation (for the age of treatment initiation with IQ and QoL) or Pearson's correlation coefficient (for FT4 with IQ and QoL).

\section{RESULTS}

The assessments of IQ and QoL were done to 25 patients with a diagnosis of congenital hypothyroidism. Almost all subjects were aged $>1$ month at the time of congenital hypothyroidism diagnosis and initiation of therapy. All patients diagnosed with congenital hypothyroidism were given therapy immediately; hence, no time lapse between diagnosis and treatment was observed. Almost all patients presented with signs of developmental delay. One patient was aged < 1 month at the time of diagnosis; hence, a developmental delay could not be determined. Patients' characteristics are described in Table 1.

As shown in Table 2, FSIQ, VIQ, and PIQ scores of all 25 children with primary congenital hypothyroidism were evaluated. Most patients scored extremely low scores (<70) for FSIQ. The lower scores of FSIQ, VIQ, and QoL were not correlated with the late initiation of treatment. Higher FT4 levels were correlated with higher scores of FSIQ and PIQ.

Overall, 19 patients agreed to undergo further investigations to determine the underlying cause of congenital hypothyroidism. The etiologies of congenital hypothyroidism determined using thyroid ultrasound or scintigraphy varied between dysgenesis (ectopic, agenesis, hypoplasia, and hemiagenesis) and
Table 2. Correlation between age of treatment initiation and FT4 level with IQ and QoL

\begin{tabular}{lcc}
\hline Components & Age at treatment initiation* & FT4 $^{+}$ \\
\hline Full-scale IQ & $(r=-0.261, p=0.071)$ & $(r=0.314, p=0.046)$ \\
Verbal IQ & $(r=-0.232, p=0.265)$ & $(r=0.341, p=0.131)$ \\
Performance & & \\
IQ & $(r=-0.325, p=0.025)$ & $(r=0.320, p=0.043)$ \\
QoL & $(r=0.006, p=0.980)$ & $(r=0.246, p=0.310)$ \\
\hline
\end{tabular}

$\mathrm{FT}_{4}=$ free thyroxine; IQ=intelligence quotient; QoL=quality of life *Kendall's Tau correlation; 'Pearson's correlation 
Table 3. Distributions of IQ results based on etiology of congenital hypothyroidism in 19 patients

\begin{tabular}{lccccc}
\hline \multirow{2}{*}{ Etiology } & \multicolumn{3}{c}{ Characteristics } & Total IQ score, mean (SD) \\
\cline { 2 - 5 } & Normal & Low average & Borderline & Intellectual disability & $37.3(8)$ \\
\hline Dyshormonogenesis $(n=3)$ & - & - & - & 3 & $46^{*}$ \\
Ectopic $(n=1)$ & - & - & - & 1 & $58.2(16)$ \\
Agenesis $(n=5)$ & - & 1 & - & 4 & $72.7(30.3)$ \\
Bilateral hypoplasia $(n=4)$ & 2 & - & - & 2 & $52.5(16.5)$ \\
Hemiagenesis $(n=6)$ & - & 1 & - & 5 & \\
\hline
\end{tabular}

$\mathrm{IQ}=$ intelligence quotient; $\mathrm{SD}=$ standard deviation

*presented in exact number

dyshormonogenesis. The most common etiology was hemiagenesis. The highest mean total IQ score was noted in the thyroid hypoplasia group. In contrast, the dyshormonogenesis group had the lowest mean total IQ score. Two patients who scored normal in the IQ tests (93 and 101) despite starting the treatment at the age of $>1$ year (at 4 and 5 years of age, respectively) had thyroid dysplasia. The distribution of IQ in each etiology type is presented in Table 3.

\section{DISCUSSION}

This study shows the importance of early diagnosis and early treatment of congenital hypothyroidism, especially in Indonesia. The number of babies born in the Asia Pacific region accounts for nearly half of the total births in the world. Around $80 \%$ of that portion is divided among the following five countries: China, Indonesia, Bangladesh, India, and Pakistan. Based on overall demographics and screened conditions of the region, Indonesia's screening coverage was still $<2 \%$. In the more developed counterparts of the Asia Pacific realm, wherein the government is fully responsible for the screening program (e.g., Australia, New Zealand, Japan, and Singapore), the percentages were already at $100 \% .^{5}$ More than a decade ago, Indonesia had started a pilot study of congenital hypothyroidism screening in two national-level hospitals in Jakarta and Bandung. By 2014, the Indonesian Ministry of Health had released a decree regarding the practice of congenital hypothyroidism screening in 14 Indonesian provinces. ${ }^{6}$ Despite this decree, insufficient financial support from the authorities in conjunction with a lack of awareness has impelled the failure of the nationwide newborn screening.

Jasin et $\mathrm{al}^{7}$ analyzed data from the hypothyroidism screening program from 2012 to 2013 to demonstrate congenital hypothyroidism characteristics among neonates in Cipto Mangunkusumo Hospital, Jakarta, Indonesia. Overall, 3,720 neonates were screened within the first 48 hours of life, whereas 14 patients were recalled for further examinations, with a recall rate of $0.38 \%$. Of the 14 patients, four were negative, one was diagnosed with Down syndrome, and nine did not comply. Notably, noncompliance is a typical facet of our participants. A delay between the time of diagnosis and treatment initiation has often occurred because of the lack of knowledge regarding the importance of timely detection and treatment of congenital hypothyroidism. ${ }^{1}$

As seen in Table 1, girls outnumbered boys (1.7:1), which is concordant with the female preponderance of congenital hypothyroidism. ${ }^{8,9}$ Overall, $96 \%$ of patients were diagnosed after the age of 1 month with a median of $9(5.5-12.5)$ years. In this study, all subjects presented with signs of developmental delay. The other clinical signs included feeding problems, constipation, umbilical hernia, and jaundice. Similarly, Rastogi and LaFranchi ${ }^{10}$ described prominent individual symptoms in congenital hypothyroidism, ranging from prolonged jaundice, lethargy, feeding difficulty to constipation.

Letarte et $\mathrm{al}^{11}$ developed a scoring system to clinically diagnose patients with congenital hypothyroidism based on 14 signs and symptoms. In developing countries, such as Indonesia, wherein the rate of newborn screening is still low, such a scoring system to diagnose congenital hypothyroidism clinically might be of merit despite the need for subsequent confirmatory tests. Congenital hypothyroidism signs and symptoms can be very subtle and are often unrecognizable. It was seen from the mean (1.5 years) and range (0.08-9.00 years) of age at treatment initiation in this study. Moreover, lapses often occur from the time of diagnosis to treatment 
initiation because of insufficient knowledge regarding the importance of timely detection and treatment of congenital hypothyroidism. ${ }^{1,5}$

The lower scores of FSIQ and VIQ were not associated with later age of treatment initiation, whereas lower scores of PIQ was associated with later age of treatment initiation. Previous study also showed that the lower scores of FSIQ, VIQ, and PIQ were not associated with treatment initiation time in patients with primary congenital hypothyroidism. ${ }^{12}$

Seo et $\mathrm{al}^{13}$ found the FSIQ, PIQ, and VIQ scores were not associated with the age at treatment initiation and initial FT4 levels. Nevertheless, in our study, the higher scores of FSIQ and PIQ were associated with higher current FT4 levels.

In this study, the late initiation of treatment and higher current FT4 levels were not correlated with health-related QoL. Meanwhile, health-related QoL in young adults previously diagnosed with congenital hypothyroidism through neonatal screening was similar to healthy individuals. ${ }^{14}$ However, other studies showed that congenital hypothyroidism increased the risk of impaired QoL. ${ }^{15}$ Long-term negative consequences of health-related QoL, development, and self-esteem are common in patients with congenital hypothyroidism. Therefore, health care providers must provide attention and offer additional emotional and educational guidance. ${ }^{16}$

Primary congenital hypothyroidism is caused by either a defect in the development of thyroid gland (dysgenesis) or defective thyroid hormone synthesis (dyshormonogenesis). Dysgenesis is the most frequent etiology that can have several forms. ${ }^{2}$ Based on thyroid ultrasonography or scintigraphy that was performed in this study, the most common etiology was also dysgenesis, especially hemiagenesis. There were two patients normal IQ scores despite starting the therapy at age $>1$ year. This might be due to thyroid hypoplasia as the etiology. Notably, another study had also observed a similar finding of normal mental ability. ${ }^{17}$

In several studies, early treatment initiation (at 12-30 days of life) have a better intellectual outcomes than late initiation (at $>30$ days of life). Upon comparing the determined etiologies of congenital hypothyroidism in that study, athyreosis was noted to be associated with the worst outcome compared with other forms of dysgenesis or dyshormonogenesis. This finding is probably due to the varied hormonal patterns among the different etiologies. ${ }^{18}$ However, limited data warrants further studies to explore the relationship between etiology and intellectual outcomes.

A recent study from Auckland suggested that early diagnosis through neonatal screening followed by an aggressive course of high dose hormonal treatment in the first 3 years leads to excellent neurocognitive and developmental outcomes in congenital hypothyroidism patients. $^{19}$ This strengthens the argument that Indonesian newborns are at a loss in achieving their maximum growth and development potential if they have congenital hypothyroidism and are neither diagnosed nor treated earlier in life.

Nonetheless, this study had some limitations. The sample size was small, and we did not correlate IQ and QoL with different etiologies of congenital hypothyroidism, bone maturation, first FT4 level, and time of normalization of FT4 and TSH levels, as well as other environmental factors. Although only one center was included in this study, patients were referred from various hospitals in Jakarta, surrounding regions, and other regions in Indonesia. Therefore, further multicenter studies are required to determine the longterm outcomes of the treatment.

Earlier detection, prompt treatment, as well as a regular follow-up may significantly improve the prognosis, developmental milestones, and QoL of patients with congenital hypothyroidism. Nevertheless, in the future, this study should motivate policymakers to implement a neonatal screening program for congenital hypothyroidism in Indonesia.

\section{Conclusions}

In summary, delayed initiation of thyroid hormone treatment in patients with congenital hypothyroidism because of the unavailability of a national newborn screening program negatively affects the intellectual abilities of affected children.

\section{Conflict of Interest}

The authors affirm no conflict of interest in this study.

\section{Acknowledgment}

The authors would like to thank Aryono Hendarto as the chairperson of Department of Child Health, Faculty of Medicine, Universitas Indonesia; Jose R. L. Batubara, Bambang Tridjaja, and Frida Soesanti from the Endocrinology Division, Department of Child Health, Faculty of Medicine, Universitas Indonesia; Tjhin Wiguna from the Department of Psychiatry, Faculty of Medicine, Universitas Indonesia; and H. F. Wulandari from Department of Child Health, Faculty of Medicine, Universitas Indonesia for their contribution and support during this study. 
Funding Sources

None.

\section{REFERENCES}

1. Rustama DS, Fadil MR, Harahap ER, Primadi A. Newborn screening in Indonesia. Southeast Asian J Trop Med Public Health. 2003;34 Suppl 3:76-9.

2. Léger J. Congenital hypothyroidism: a clinical update of longterm outcome in young adults. Eur J Endocrinol. 2015;172(2):R6777.

3. Klein AH, Meltzer S, Kenny FM. Improved prognosis in congenital hypothyroidism treated before age three months. $J$ Pediatr. 1972;81(5):912-5.

4. Rahmani K, Yarahmadi S, Etemad K, Koosha A, Mehrabi Y, Aghang $\mathrm{N}$, et al. Congenital hypothyroidism: optimal initial dosage and time of initiation of treatment: a systematic review. Int J Endocrinol Metab. 2016;14(3):e36080.

5. Therrell BL, Padilla CD, Loeber JG, Kneisser I, Saadallah A, Borrajo $G J$, et al. Current status of newborn screening worldwide: 2015. Semin Perinatol. 2015;39(3):171-87.

6. Indonesian Ministry of Health. Ministreal Decree no.78 issued on 2014. [statute on the Internet]. 2017 [cited 2017 Oct 30]. Available from: http://kesga.kemkes.go.id

7. Jasin MR, Prasmusinto D, Pulungan AB. Epidemiology of congenital hypothyroidism and screening program in Dr. Cipto Mangunkusumo hospital: a hospital-based study. $6^{\text {th }}$ Annual Scientific Meeting of Indonesian Pediatric Society 2013. 2013 Oct 5-9; Solo (Indonesia). c2017.

8. Rezaeian S, Moghimbeigi A, Esmailnasab N. Gender risk factors of congenital hypothyroidism: an interaction hypothesis examination. Int J Endocrinol Metab. 2014;12(2):e13946.

9. Waller DK, Anderson JL, Lorey F, Cunningham GC. Risk factors for congenital hypothyroidism: an investigation of infant's birth weight, ethnicity, and gender in California, 1990-1998. Teratology. 2000;62(1):36-41.

10. Rastogi MV, LaFranchi SH. Congenital hypothyroidism. Orphanet
J Rare Dis. 2010;5:17.

11. Letarte J, Guyda H, Dussault JH. Clinical, biochemical and radiological features of neonatal hypothyroid infants. In: Burrow GN, ed. Neonatal thyroid screening. New York: Raven Press. 1980. p. 225-35.

12. Najmi SB, Hashemipour M, Maracy MR, Hovsepian S, Ghasemi $M$. Intelligence quotience in children with congenital hypothyroidism: the effect of diagnostic and treatment variables. J Res Med Sci. 2013;18(5):395-9.

13. Seo MK, Yoon JS, So CH, Lee HS, Hwang JS. Intellectual development in preschool children with early treated congenital hypothyroidism. Ann Pediatr Endocrinol Metab. 2017;22(2):102-

14. Sato H, Nakamura N, Harada S, Kakee N, Sasaki N. Quality of life of young adults with congenital hypothyroidism. Pediatr Int. 2009;51(1):126-31.

15. van der Sluijs Veer L, Kempers MJ, Maurice-Stam H, Last BF, Vulsma T, Grootenhuis MA. Health- related quality of life and selfworth in 10-year old children with congenital hypothyroidism diagnosed by neonatal screening. Child Adolesc Psychiatry Ment Health. 2012;6(1):32.

16. van der Sluiis Veer L, Kempers MJ, Last BF, Vulsma T, Grootenhuis MA. Quality of life, developmental milestones, and self-esteem of young adults with congenital hypothyroidism diagnosed by neonatal screening. J Clin Endocrinol Metab. 2008;93(7):265461.

17. Mendorla G, Sava L, Calaciura F, Lisi E, Castorina S, Vigneri R. Personality traits and mental prognosis in patients with congenital hypothyroidism not treated from early life. J Endocrinol Invest. 1988;11(4):289-95.

18. Hanukoglu A, Perlman K, Shamis I, Brnjac L, Rovet J, Daneman D. Relationship of etiology to treatment in congenital hypothyroidism. J Clin Endocrinol Metab. 2001;86(1):186-91.

19. Albert B, Heather N, Cutfield W, Webster D, Gunn A, Jefferies $C$, et al. Neurodevelopmental outcomes are normal in congenital hypothyroid children diagnosed early and treated aggressively over the first three years. Int J Pediatr Endocrinol. 2013;2013(Suppl 1):023. 